\title{
Nanotechnologie: Eine neue soziale Dynamik an der Schnittstelle zwischen Wissenschaft und Öffentlichkeit
}

Joachim Schummer

Nanotechnology: New Social Dynamics at the Interface between Science and the Public

This paper investigates the development of nanotechnology from three different points of view: (1) as a new technology, (2) as social dynamics, and (3) as an ideology. It argues that nanotechnology is not a new technology but a new social dynamics guided by programmatic ideas and situated at the interface between science and the public. Rather than being determined by social constructivism, the main argument is based on the poor scientific and technological identity of nanotechnology. Finally the paper concludes that nanotechnology is to be considered an extension and increase of materials science and engineering, not only scientifically but in particular socially and ideologically, by pointing out common characteristics and differences.

Keywords: nanotechnology, new technology, social dynamics, ideology, materials science and engineering

Schlüsse/wörter: Nanotechnologie, neue Technik, soziale Dynamik, Ideologie, Materialwissenschaften

In den 1990er Jahren, als man das Ende des Jahrhunderts oder sogar des Jahrtausends ins Auge fasste, zeigte sich weltweit ein Bedürfnis nicht nur nach Bestandsaufnahme der jüngeren Vergangenheit, sondern vor allem auch nach Ausblick in die Zukunft einer neuen Epoche. Wie in allen Bereichen so wurde auch in Wissenschaft und Technik darum gerungen, wer den hellsichtigsten Blick in die Zukunft wirft und die Technik des 21. Jahrhunderts oder gar des dritten Jahrtausends voraussieht. In keinem anderen Land wurde dieses Bedürfnis nach Prophezeiung einer neuen Epoche so ausgiebig ausgelebt wie in den USA, wo dies im sogenannten Dispensionalimus eine starke religiöse Tradition besitzt.

In den zehn Jahren seit dem Jahrtausendwechsel hat sich die Nanotechnologie von einer Marginalie der Wissenschaft zu einer globalen Hauptströmung entwickelt (Schummer 2007). Schaut man hingegen in die professionellen 
Prognosen der 1990er Jahre, dann stellt man überraschend fest, dass bis etwa 1999 kaum jemand von ihrem Kommen ahnte. Zum Beispiel organisierte die American Chemical Society eine Tagung mit dem vielversprechenden Titel "Chemical Research - 2000 and Beyond: Challenges and Visions“, zu dem die wichtigsten chemischen Honoratioren des Landes beitrugen und deren Beiträge als Sammelband veröffentlicht wurden (Barkan 1998). Doch während Nanotechnologie heute gerade bei den amerikanischen Chemikern zum Hauptgebiet avancieren konnte, sucht man das Thema damals in dem vermeintlich visionären Ausblick vergeblich. Die amerikanischen Akademien der Technik und der Wissenschaft organisierten sogar fächerübergreifend jeweils jährliche Symposien zu „Frontiers of Engineering“ und „Frontiers of Science“, doch Nanotechnologie wurde dort erstmals im Jahr 2000 beziehungsweise 2001 erwähnt. ${ }^{1}$

Man könnte versucht sein, die Unvorhersehbarkeit der Nanotechnologie durch die schwierige Prognostizierbarkeit von Forschung im allgemeinen zu begründen, da diese sich plötzlich durch überraschende Entdeckungen oder Erfindungen in ungeahnte Richtungen entwickeln kann. Doch von solchen überraschenden Entdeckungen und Erfindungen, die Nanotechnologie auf die Wege gebracht haben könnten, ist um das Jahr 2000 gar nichts bekannt. Das lässt vermuten, dass die Entstehung und der ungewöhnliche Erfolg der Nanotechnologie anderen, eher außerwissenschaftlichen Einflüssen zu verdanken sind.

Dieser Beitrag untersucht die Entwicklung der Nanotechnologie unter drei unterschiedlichen Gesichtspunkten, nämlich als neue Technik, als soziale Dynamik und als programmatische Idee. ${ }^{2}$ Ich argumentiere, dass Nanotechnologie keine historisch neue Technik ist, sondern eine von programmatischen Ideen geleitete neue soziale Dynamik an der Schnittstelle zwischen Wissenschaft und Öffentlichkeit. Das Ergebnis wird dabei nicht von einer vorausgesetzten sozialkonstruktivistischen Perspektive, sondern durch die ausgesprochene Schwäche der wissenschaftlich-technischen Inhaltsebene bestimmt. Abschließend zeige ich, dass Nanotechnologie in diesem Sinne als eine Weiterführung der Materialwissenschaften verstanden werden kann, indem ich Gemeinsamkeiten und Unterschiede zwischen beiden Entstehungsprozessen aufzeige.

\section{Nano als neue Technik}

Die allgemein übliche Rede von „der Nanotechnologie“ legt nahe, dass Nanotechnologie eine einheitliche und neue Technik sei, die sich begrifflich klar von anderen Techniken unterscheiden lässt. Tatsächlich gaben die Gründer der amerikanischen National Nanotechnology Initiative (NNI) eine Nominaldefinition vor, die von späteren Nano-Initiativen fast aller anderen 
Länder übernommen wurde und sich seitdem weitgehend behaupten konnte. Sie definierten Nanotechnologie als:

„Forschung und technische Entwicklung auf der atomaren, molekularen oder makromolekularen Ebene im Längenbereich von ungefähr 1-100 Nanometern, um ein grundlegendes Verständnis von Phänomenen und Materialien im NanoBereich zu liefern und um Strukturen, Geräte (devices) und Systeme zu erzeugen und zu gebrauchen, die neue Eigenschaften und Funktionen aufweisen wegen ihrer kleinen Größe und/oder Zwischengröße." (National Science and Technology Council 2000, meine Übersetzung)

An dieser Definition fällt auf, wie weit sie gefasst ist, da bekanntermaßen unter Normalbedingungen alle kondensierte Materie ,auf der atomaren, molekularen oder makromolekularen Ebene im Längenbereich von ungefähr 1-100 Nanometern" strukturiert ist. Selbst die meisten klassischen Festkörper besitzen eine kristallographische Einheitszelle, die mindestens in einer Dimension im Bereich von 1-100 Nanometern liegt. Und unter den etwa fünf Millionen neuen molekularen und makromolekularen Substanzen, die der Chemical Abstract Service jährlich registriert (CAS 2008), dürfte aus molekularer Perspektive kaum etwas außerhalb des „Nano-Bereichs“ liegen. Selbstverständlich lässt sich auch jedes Molekül oder Makromolekül als System oder Gerät (device) betrachten, sobald man eine systemische beziehungsweise funktionale Perspektive anlegt. Da also nach bester wissenschaftlicher Überzeugung fast alle kondensierte Materie im „Nano-Bereich“ strukturiert ist und ihre Eigenschaften, einschließlich ihrer systemischen, funktionalen oder technisch verwertbaren Eigenschaften, durch diese Strukturen wesentlich bestimmt werden, fällt unter die Definition der Nanotechnologie fast jede Wissenschaft und Technik, die sich mit materiellen Gegenständen und ihren Eigenschaften beschäftigt. Dazu gehören insbesondere seit Mitte des 19. Jahrhunderts fast der gesamte Bereich der Chemie und seit etwa Mitte des 20. Jahrhunderts große Teile der Pharmazie, Molekularbiologie, Festkörperphysik und Elektrotechnik sowie selbstverständlich die gesamten Materialwissenschaften. Die gängige Definition der Nanotechnologie ist daher so weit, dass sie kaum etwas Spezifisches und jedenfalls nichts Neues bestimmt und stattdessen Bereiche aus fast jeder klassischen Disziplin umfasst.

Der definitorischen Schwäche der Nanotechnologie wurde aus verschiedenen Richtungen begegnet. Natürlich gab es begriffliche Präzisierungsversuche, die sich jedoch nicht durchsetzen konnten - manchmal nicht einmal bei den Autoren. ${ }^{3}$ Auch wird gerne darauf hingewiesen, dass zu Beginn alles Neue, alles im Entstehen Befindliche sich klarer begrifflicher Einordnung widersetzt. Doch solange dies der Fall ist, ist auch noch nicht ausgemacht, um was es sich dabei eigentlich handelt und ob es dabei überhaupt um etwas Zusammenhängendes und Neues geht. Ebenfalls sind die verbreiteten Sonntagsreden von der Querschnittstechnologie und der Schlüsseltechnologie die 
Nachweise schuldig geblieben, dass die Nanotechnologie tatsächlich eine Querverbindung zwischen den Disziplinen schafft und ein Schlüssel für verschiedene weitere Zukunftstechnologien ist. Letzteres wird gerne für jede in der Entwicklung befindliche Technik behauptet, weil es deren technische Bedeutung mit Bezug auf eine unbestimmte Zukunft aufwertet und daher zunächst nur als Marketingstrategie zu werten ist. Demgegenüber lässt sich empirisch untersuchen, ob Nanotechnologie tatsächlich eine Verbindung quer zu den Disziplinen herstellt. Dass Interdisziplinarität einschließlich einer verstärkten Zusammenarbeit zwischen Natur- und Technikwissenschaften ein wichtiges Ziel der Wissenschaftspolitik ist, lässt sich in jeder wissenschaftspolitischen Broschüre zur Nanotechnologie nachlesen (siehe unten). Die Frage ist jedoch, ob Nanotechnologie dieses Ziel tatsächlich erfüllt oder ob sie umgekehrt eher als ein wissenschaftspolitisches Instrument zur Erfüllung des Zieles zu verstehen ist.

Zwar haben sich heute alle natur- und technikwissenschaftlichen Disziplinen „Nano“ auf ihre Fahnen geschrieben, aber ein gemeinsames Etikett, das die Aussicht auf Forschungsförderung erhöht, garantiert noch keine Interdisziplinarität, sondern allenfalls Multidisziplinarität. Was heute unter Nanotechnologie läuft ist fast so multidisziplinär, wie es die Wissenschaft selbst ist. Untersucht man hingegen die interdisziplinäre Zusammenarbeit der nano-etikettierten Forschung, etwa durch Koautoren-Analyse von Aufsätzen in vermeintlich interdisziplinären Nanotechnologie-Zeitschriften, dann zeigt sich kaum ein besonderer Grad von Interdisziplinarität. Vielmehr scheint jede der klassischen Disziplinen ihre eigenen Nanotechnologien zu pflegen und über eigene, überwiegend monodisziplinäre Nano-Zeitschriften zu kommunizieren (Schummer 2004a).

Wenn daher Nanotechnologie weder eine begrifflich klar fassbare neue Technologie noch eine strukturelle Neuheit im Sinne einer neuen Querschnittstechnologie ist - lässt sich Nanotechnologie dann zumindest als ein bestimmtes Bündel neuer Einzeltechnologien auffassen, die zusammengenommen den Kriterien einer Realdefinition genügen könnten? Tatsächlich hat jede klassische Disziplin heute eine lange Liste von Forschungsgebieten als Nanotechnologie vereinnahmt, die jedoch von Land zu Land variiert und im Laufe der Zeit stetig gewachsen ist, so dass hier nur einige Gebiete beispielhaft genannt werden können. In Chemie, Materialwissenschaft und chemischer Technik zählen dazu typischerweise die Forschung und Entwicklung von Katalyse, „molekulare Maschinen“, Synthese durch „Selbstorganisation“, Polymere und Nanoverbundstrukturen, Flüssigkristalle, quantenchemische Modelrechnungen und molekulardynamische Simulationen, Kohlenstoffnanoröhrchen, ultradünne Beschichtungen und molekulare Filme, nanostrukturierte Festkörper, Nanopartikel, Wasserstoffspeichersysteme und Brennstoffzellen und so weiter. Physik, Elektrotechnik und Metrologie favorisieren insbesondere Rastersonden- und Elektronenmikroskopie sowie die gesamte Palette von Techniken zur elektronischen und optischen Speicherung 
und Verarbeitung von Daten, einschließlich Halbleiterdotierung und lithographischer Verfahren der Chip-Produktion. In Deutschland erhielt anfangs kurioserweise auch die Laserforschung das Nano-Etikett. Der biomedizinische Bereich positioniert unter „Nanotechnologie“ (neuerdings auch unter „Bionanotechnologie“, „Nanobiotechnologie“ oder „Nanomedizin“) besonders gerne drug delivery systems (also molekulare Verpackungssysteme, die Wirkstoffe durch den Körper schleusen und lokalisiert freisetzen), biochemische Sensoren und bildgebende Verfahren in der Medizin sowie zunehmend auch Bereiche der Neurophysiologie und Gewebetechnik. Da die Einbeziehung der klassischen Gentechnik aus gesellschaftlichen Gründen problematisch ist, wird sie teilweise unter dem Stichwort synthetic biology in den Bereich der Nanotechnologie integriert, wozu auch diverse andere Methoden der Modifikation und de novoSynthese von biologischen Zellelementen gehören.

Angesichts der Vielzahl und Heterogenität von Forschungsgebieten der Nanotechnologie, deren Liste sich fast beliebig verlängern ließe, erscheint es schwierig, einen Überblick zu gewinnen, der zudem wissenschaftshistorisch informiert ist. Tatsächlich lassen sich aber für fast alle diese Forschungsgebiete längere Traditionen nachweisen, die bis weit ins 20. Jahrhundert und oft darüber hinaus zurückreichen. Einige Beispiele mögen das illustrieren.

Die chemische Katalyse wurde seit dem frühen 20. Jahrhundert systematisch untersucht und großindustriell umgesetzt, einschließlich beispielsweise der Verwendung synthetischer Zeolithe mit maßgeschneiderten Poren im Nanometerbereich seit den 1950er Jahren (Sherman 1999). Die Geschichte quantenchemischer Modellrechnungen begann in den 1920er Jahren und entwickelte sich seit Mitte des Jahrhunderts zusammen mit molekulardynamischen Simulationen weitgehend parallel $\mathrm{zu}$ den Verbesserungen der Computertechnik (Simões/Gavroglu 2001, Peyerimhoff 2002). Kohlenstoffnanoröhrchen (CNT), die inzwischen zu graphischen Ikonen der Nanotechnologie geworden sind, wurden bereits 1952 hergestellt, untersucht und durch Transmissionselektronenmikroskopie charakterisiert (Monthioux/Kuznetsov 2006). Chemische Gasphasenabscheidung war bereits im 19. Jahrhundert bekannt und wurde seit den 1960er Jahren zur Herstellung von mikro- und nanoskaligen Filmen (für ultradünne Beschichtungen einschließlich solcher für die Halbleiterlithographie) sowie von Nanopartikeln und dotierten Halbleitern verwendet (Allendorf 1998). Die Herstellung molekularer Geräte oder „Maschinen“ - bis vor kurzem noch supramolekulare Chemie genannt begann etwa zeitgleich mit der Gentechnik in den 1970er Jahren (Lehn 1992, Balzani/Credi/Venturi 2003). Während die Elektronenmikroskopie seit den 1920er Jahren fortentwickelt wurde, startete die Geschichte der Rastersondenmikroskopie in den frühen 1980er Jahren (Mody 2004, Baird/Shew 2004). Ein Blick in Patentdatenbanken zeigt außerdem, dass Wasserstoffspeichersysteme, drug delivery systems (zum Beispiel mit Liposomen, Polymeren oder Cyklodextrinen) und viele andere Systeme, die heute unter 
Nanotechnologie rangieren, seit den frühen 1970er Jahren nicht nur intensiv untersucht, sondern auch umfangreich patentiert wurden - wobei übrigens die großen, heute wiederkehrenden Hoffnungen bei den drug delivery systems oft erst während der klinischen Studien zerbrachen. ${ }^{4}$ Für die meisten Systeme der Nanotechnologie - einschließlich Nanopartikeln und Kolloiden (Woyke 2008, Ede 2007), Flüssigkristallen (Kelker 1973, Kawamoto 2002), Polymeren (Furukawa 1998), Nanocomposites (Bensaude-Vincent 1998), nanostrukturierten Materialien (Nordmann 2006), Nanofilmen (Roberts 1990: Kap. 1) und so weiter - gilt, dass sie über weite Zeiträume des 20. Jahrhunderts hergestellt und systematisch untersucht worden sind, wobei man oft auf Forschungen des 19. Jahrhunderts sowie auf frühere handwerkliche Praktiken und Produkte zurückgreifen konnte.

Damit können wir eine erste Zwischenbilanz ziehen: Auf wissenschaftlicher Ebene verbirgt sich hinter der Nanotechnologie weder etwas inhaltlich oder strukturell Einheitliches, das einen gemeinsamen Namen rechtfertigen könnte, noch etwas grundlegend Neues. Freilich produziert jeder einzelne der oben genannten Forschungsbereiche beständig Neues, weil dies eine Grundbedingung jeder wissenschaftlichen Forschung ist. Es scheint sogar, dass zur Nanotechnologie besonders spannende und ökonomisch erfolgversprechende Bereiche gezählt werden, die durch zusätzliche Budgets sicherlich mehr Neues produzieren werden. Doch das begründet alleine weder eine technologische Gemeinsamkeit noch eine neue Technologie. Neu scheint lediglich die Verwendung der Einheit Nanometer zur Beschreibung materieller Gegenstände zu sein, für die man bis zum Jahr 2000 in der Wissenschaft aus historischen Gründen die Einheiten Ángström, Mikrometer und Kilodalton verwendete, was einen großen Raum für Umetikettierung eröffnete.

Der Mangel an brauchbaren Definitionen, der Schein einer neuen Vereinigung aller Disziplinen und die implizite Geschichtsvergessenheit mit Bezug auf wissenschaftliche Forschungstraditionen legen alle nahe, dass die Rede von der Neuheit der Nanotechnologie eher sozialen Bedürfnissen als dem Bemühen um begriffliche Klarheit entsprungen ist. Und sie zeigen klar, dass mit der Rede von Nanotechnologie primär ein außerwissenschaftliches Publikum angesprochen wird, das an Wissenschaft lediglich allgemein interessiert ist, ohne weiter reichendes Verständnis von Forschungsdetails und historischen Traditionen zu besitzen. Welcher Wissenschaftler wollte die begriffliche Beliebigkeit und die Umetikettierung etablierter Forschungsbereiche anprangern, wenn sie ihm im Nanohype zu ungeahntem Geldsegen verhelfen?

\section{Nano als soziale Dynamik}

Nicht eine sozialkonstruktivistische Perspektive, sondern der Mangel an Kriterien auf wissenschaftlich-technischer Ebene führt uns zwangsläufig dazu, 
Nanotechnologie als sozialen Prozess genauer zu analysieren. Dass hierbei die Wissenschaftspolitik eine entscheidende Rolle einnimmt, wird deutlich an der Tatsache, dass die verbreitete Rede von Nanotechnologie in der Wissenschaft mit der Gründung der National Nanotechnology Initiative (NNI) der USA durch den damaligen Präsident Clinton pünktlich zur Jahrtausendwende im Januar 2000 einsetzte.

Bevor es jedoch weltweit nationale Nanotechnologie-Initiativen gab, wurden die wissenschaftspolitischen Argumente für eine solche Initiative entwickelt. Eine zentrale Argumentationsfigur bezog sich dabei auf konkurrierende Forschungsbudgets, die noch im Geiste des Kalten Krieges zwar nicht mehr Russland anvisierte, aber doch die Welt in drei vermeintliche Konkurrenten aufteilte - die USA, Europa und Japan -, die mit ihren Nano-Budgets in etwa gleicher Größenordnung um die Vorherrschaft ringen würden. ${ }^{5}$ Diese imaginierte Forschungskonkurrenz dominierte zumindest in den USA noch mindestens bis 2005, obwohl China und Südkorea schon damals wissenschaftliche Führungsrollen übernommen hatten, die interessanterweise wegen der geringen Lohnkosten von Wissenschaftspolitikern schlichtweg übersehen wurden (Schummer 2007). Dieser Befund sowie die zum Teil bis heute noch vorherrschende Praxis, Nanotechnologie in Dollar-Input zu messen, legen nahe, dass es bei der Nanotechnologie gar nicht um Technologie oder Forschungsoutput ging, sondern um Geld, genauer gesagt um nominale Forschungsbudgets in einem imaginierten internationalen Wettbewerb, der als ein symbolisches Echo des Kalten Krieges erscheint.

In diesem Wettbewerb ist der Mangel an begrifflich klaren Definitionen von Nanotechnologie von Beginn an durchaus willkommen gewesen, da man so durch fast beliebige Zuordnung die Größe der Budgets und damit die eigene Führungsrolle in der Nanotechnologie gestalten konnte. Das lässt sich sehr gut am Beispiel des deutschen Bundesministeriums für Forschung und Technologie (BMBF) illustrieren. Dieses publizierte im Januar 2002 seinen Faktenbericht Forschung 2002 (BMBF 2002a), in dem die gesamten Forschungsausgaben des Bundes nach Förderbereichen und -programmen detailliert aufgeschlüsselt wurden. Darin findet sich „Nanotechnologie“ noch als Unterbereich von "Materialforschung; physikalische und chemische Technologien“ mit einem Gesamtfördervolumen von knapp 72 Millionen Euro für den Zeitraum 1997-2005 (ebd.: 269). Dies entsprach einem jährlichen Budget von durchschnittlich 8 Millionen Euro oder einem Promille des damaligen Jahresetats des BMBF. Wenige Monate später, im Juni 2002, nachdem die Europäische Kommission in ihrem neuen Rahmenprogramm Nanotechnologie zum Schwerpunktthema erhoben hatte, gab das gleiche Ministerium die Broschüre Nanotechnologie in Deutschland: Standortbestimmung heraus (BMBF 2002b). Darin bezifferte das BMBF allein seine eigenen Förderausgaben für Nanotechnologie mit 350 Millionen Euro für den engeren Zeitraum 1998-2004, was einem jährlichen Budget von durchschnittlich 50 Millionen Euro entspricht (ebd.: 15). 
Dabei wurden rückwirkend nicht nur die Budgets der 1990er Jahre aufgestockt, um frühen forschungspolitischen Weitblick zu demonstrieren; man baute auch rückwirkend einen gehörigen Zuwachs ein, so dass man stolz verkünden konnte: „Die derzeitige BMBF-Förderung der Nanotechnologie ist seit 1998 um 221\% angestiegen." ${ }^{\text {“6 }}$

Im Unterschied zu den beliebig konstruierten und rekonstruierten NanoBudgets der frühen Phase, erhielt die Nanotechnologie nach 2000 in vielen Ländern ein fest zugeordnetes staatliches Forschungsbudget in Gestalt von nationalen Initiativen oder Programmen. Was inhaltlich zur Nanotechnologie zählte, wurde insbesondere durch diese Nano-Budgets und ihre Verteilungsschlüssel festgelegt, auch wenn hier von Land zu Land oft beträchtliche Unterschiede auftraten, die erst durch das alles einverleibende Wachstum der Nanothemen und -budgets nivelliert wurden. Die soziale Geschichte der Nanotechnologie ist daher hauptsächlich eine Geschichte der wissenschaftspolitischen Nanoprogramme und ihrer Budgets.

Diese Geschichte begann bereits mit dem 1992 gegründeten Atomtechnologieprojekt des Japanischen Industrie- und Handelsministeriums, das „die Erforschung und Entwicklung der Manipulation von Atomen und Molekülen" zum Ziel hatte (National Science and Technology Council 1999: 256-260, meine Übersetzung). Das Projekt umfasste ursprünglich ein breites Spektrum von Grundlagen- und angewandter Forschung der Chemie und Physik und wurde später durch Komponenten aus Elektrotechnik, Materialwissenschaften, Chemieingenieurwesen und Maschinenbau erweitert. Es lief im Stile japanischer Planwirtschaft zehn Jahre, bis es, nach Gründung der NNI, als Nanotechnologieprojekt weiterleben und wachsen durfte. Noch bis Anfang des nächsten Jahrzehnts blieben die Ausdrücke „nanotechnology“ und ,atom technology" weitgehend austauschbar, so dass beispielsweise die kanadische ETC-Gruppe selbst noch 2003 die Nanotechnologie unter dem Stichwort „atom technology“ kritisierte (ETC Group 2003).

Kurioserweise entstand das von den Amerikanern zunächst so argwöhnisch beobachtete japanische Atomtechnologieprojekt auch auf Druck der USA, die den Japanern seit Jahren vorgeworfen hatten, eigene Grundlagenforschung zu vernachlässigen und stattdessen ihre Industrieforschung auf fremde Resultate $\mathrm{zu}$ stützen (Fujita 2005). In den Berichten zur Vorbereitung der NNI wurde dann aber umgekehrt auf die wachsende Forschungskonkurrenz aus Japan (und Europa) verwiesen, insbesondere auf deren fiktive Nano-Budgets, um die eigene Forschungsinitiative politisch zu rechtfertigen. Entsprechend zeigten später auch alle europäischen Berichte auf die amerikanischen und japanischen Budgets, um zu Hause neue Forschungsgelder und -programme für Nanotechnologie zu legitimieren. So wurde Nanotechnologie in zirkulären Verweisen als forschungspolitische Leitidee international hochgeschaukelt (Fogelberg/Glimell 2003: 47).

Der eigentliche Startschuss fiel jedoch erst mit der Gründung der amerikanischen NNI im Januar 2000. In den Folgejahren entstanden nationale 
Forschungsprogramme für Nanotechnologie nicht nur in traditionell forschungsstarken Industrienationen wie in Kanada und Israel (2001) sowie in der Europäischen Union (2002), ${ }^{7}$ sondern auch in Schwellen- und Entwicklungsländern wie in Brasilien, Südkorea, Indien und Singapur (2001), in China und Taiwan (2002) und in Südafrika, Thailand und im Iran (2003). ${ }^{8}$

Im Rückblick erscheint die Entwicklung der wissenschaftspolitischen Idee der Nanotechnologie fast wie ein Prozess spontaner Selbstorganisation auf globaler Ebene. Dessen Initialzündung war die Gründung der NNI, teilweise als Reaktion auf das von den USA mitveranlasste und gerne missverstandene japanische Atomtechnologieprojekt. Analog zum Wettrüsten des Kalten Krieges begann ein Wettlauf um das größte Nano-Budget, dem sich bald auch Europa und zahlreiche Industrie-, Schwellen- und Entwicklungsländer jeweils in ihrer Liga in anschlossen. Mit einiger Verspätung gesellte sich übrigens Russland erst 2007 mit der Ankündigung eines Fünf-Milliarden-DollarProjekts, „Rosnanotekh“ dazu, was fast der Höhe des gesamten sonstigen zivilen Wissenschaftsetats Russlands entspricht (Putin 2007). Binnen weniger Jahre sind die ursprünglichen Budgets auf ein Vielfaches, oft auf das Hundertfache, angewachsen. Für die wissenschaftspolitische Idee der Nanotechnologie blieb es dabei unbedeutend, ob die Zuwächse durch Umverteilung oder durch Begriffserweiterung geleistet wurden.

Der wissenschaftspolitische Aktionismus übte von Beginn an einen starken Einfluss auch auf die Wissenschaft selbst aus, was sich zunächst jedoch hauptsächlich in veränderten Sprachgepflogenheiten zeigte. Traditionelle Einheiten wie das Ångström wichen dem Nanometer - und zwar etwa proportional zum Nano-Budget, wie sich an den gut dokumentierten Daten der USA zeigen lässt. In Abbildung 1 wird die Verwendung des Präfixes „nano“ im Titel wissenschaftlicher Aufsätze aus den USA mit dem Nano-Budget der National Science Foundation verglichen, die den Großteil der Forschung in den USA finanziert. ${ }^{9}$ Man sieht hier zunächst ein stetiges aber langsames Anwachsen der Aufsätze mit „nano“ im Titel über die gesamten 1990er Jahre,

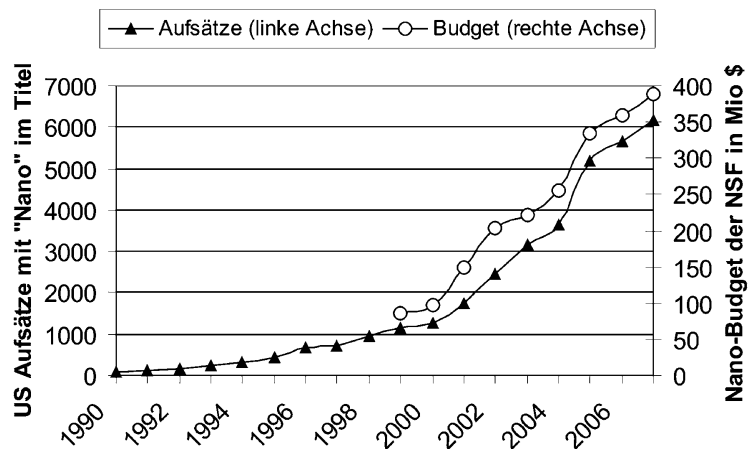

Abb. 1 Vergleich der Anzahl der Aufsätze mit „nano“ im Titel von Autoren mit dem NanoBudget der US-amerikanischen National Science Foundation 
was weitgehend auf optischer Forschung im Nanometerwellenlängenbereich basiert sowie auf älteren Projekten zur Mikro- und Nanofabrikation in der Halbleitertechnik. Ab 2000, also mit Beginn der NNI, steigt die Kurve plötzlich stark an und verläuft dann fast gleichförmig mit den verschiedenen Schwankungen im Budget. Als zum Beispiel der Zuwachs des Nano-Budgets 2003 bescheidener ausfiel, wurden auch die Wissenschaftler weniger enthusiastisch im Gebrauch von „nano“, bis der starke Budgetanstieg ab 2004 ihre Wortwahl wieder beflügelte. Mit den erneut dürftigeren Budgetzuwächsen seit 2006 scheint jedoch auch der sprachliche Enthusiasmus wieder etwas abgeflaut zu sein. Der nahezu parallele Verlauf der beiden Kurven illustriert sehr schön, wie die Wissenschaft mit unverbindlichen Umetikettierungen dem Geld der Wissenschaftspolitik gefolgt ist.

Während die Verwendung von „nano“ in Aufsatztiteln kaum mehr als ein Lippenbekenntnis sein muss, haben sich Wissenschaftler aber auch zunehmend zur Nanotechnologie als einer mittel- bis längerfristigen Entwicklung bekannt, indem sie sich beispielsweise an deren Institutionalisierung beteiligten. Dazu gehört etwa die Gründung neuer Fachzeitschriften, die explizit der Nanotechnologie gewidmet sind. Indem sich Wissenschaftler sowohl als Herausgeber als auch als Autoren am Aufbau einer neuen Nano-Zeitschrift beteiligen, investieren sie im Sinne ihrer eigenen Kariere in das Gebiet und bekennen sich zu dessen Zukunftsfähigkeit. Gleiches gilt erst recht für Verlage und wissenschaftliche Gesellschaften, wenn sie eine neue Nano-Zeitschrift verlegen. Daher sind Zeitschriftengründungen wichtige Frühindikatoren für die Institutionalisierung eines neuen Gebiets. Bis Mitte 2008 wuchs die Anzahl der „Nano-Zeitschriften“ auf etwa 50 an, wobei in der Anfangsphase NanoZeitschriften eher von wissenschaftlichen Gesellschaften gegründet wurden, wie NanoLetters (seit 2001) von der American Chemical Society und Transactions of Nanotechnology (seit 2002) vom Institute of Electrical and Electronics Engineers. Demgegenüber hielten sich die großen kommerziellen Wissenschaftsverlage zunächst zurück und positionierten ihre NanoZeitschrift erst in einer zweiten Gründungswelle, wie Wiley mit Small (seit 2005), die Nature Publishing Group mit Nature Nanotechnology (seit 2006) und Springer mit Nanoscale Research Letters (seit 2007).

Ein weiterer Indikator der Institutionalisierung ist die Gründung neuer akademischer Institutionen, die „nano“ im Namen tragen. Solche NanoInstitutionen haben sich in den letzten zehn Jahren mit einer bisher unbekannten Geschwindigkeit global vermehrt mit jährlichen Wachstumsraten von über 50 Prozent (Schummer 2007). Der Prozess fand hauptsächlich an Universitäten und weniger an staatlichen oder privaten Forschungseinrichtungen statt, so dass heute fast jede Universität weltweit mindestens eine Nano-Institution besitzt und der Institutionalisierungsgrad vergleichbar erscheint mit dem von alten Disziplinen wie Physik oder Chemie. Allerdings resultiert der Großteil dieser Nano-Institutionen aus der Umbenennung 
etablierter Institutionen oder besteht aus virtuellen Netzwerken und losen Assoziationen. Davon muss man die eigentlichen institutionellen Neugründungen unterscheiden, die in die bestehenden institutionellen Strukturen fest verankert werden. Aber auch bei den kleineren Arbeitsgruppen oder losen Assoziationen zur Nanotechnologie zeigt sich in vielen Ländern der klassische Weg disziplinärer Institutionalisierung, indem schrittweise größere und fester verankerte Institutionen bis zur Department- oder Fakultätsebene wachsen, mit eigenem Budget, eigener Verwaltung und inzwischen sogar eigenen Studienprogrammen.

Die Einrichtung neuer Nanostudiengänge, wenn sie unabhängig oder quer zu den etablierten Disziplinen angeboten werden, ist vielleicht der weitestreichende Schritt und stärkste Indikator für die Institutionalisierung von Nanotechnologie. Zumindest gilt für die heutigen Studenten, dass ihnen damit Karrieremöglichkeiten in den klassischen Disziplinen erheblich beschnitten werden, so dass sie, ob sie es wollen oder nicht, die Institutionalisierung der Nanotechnologie schon aus Existenzgründen in der weiteren Zukunft vorantreiben müssen.

Wir haben bisher gesehen, dass Nanotechnologie zwar weder eine einheitliche Technik noch auf wissenschaftlicher Ebene eine Neuheit ist, jedoch als soziale Bewegung durchaus neu und ernst zu nehmen ist. Die Nanotechnologie-Initiative der USA hat über weltweite Nachahmer und die imaginierte Konkurrenz von Forschungsbudgets eine globale wissenschaftspolitische Bewegung ausgelöst, welche die Nanotechnologie innerhalb weniger Jahre von der Nichtexistenz zum internationalen Förderschwerpunkt erhoben hat. Gleichzeitig hat sich auch der Einfluss auf die eigentliche Wissenschaft erheblich verändert. Aus der ursprünglich unverbindlichen Anpassung von Sprachgewohnheiten, die noch als vorübergehende Mode erscheinen konnte, wurde ein Prozess der (Re-)Institutionalisierung von Wissenschaft, der quer zu fast allen Disziplinen läuft, sich mit beispielloser Geschwindigkeit fortsetzt und globale Ausmaße besitzt, so dass er kaum noch umkehrbar erscheint. Um zu verstehen, wozu diese gewaltige (Re-)Institutionalisierung von Wissenschaft stattfindet, müssen wir betrachten, welche Ideen mit Nanotechnologie von wem verfolgt werden.

\section{Nanotechnologie als Ideologie}

Solange Nanotechnologie begrifflich unscharf bis diffus bleibt, bietet sie eine Projektionsfläche allgemeiner Technikaffekte, von Heilserwartungen bis zu existentiellen Ängsten. Daher eignen sich solche Projektionsflächen umgekehrt auch hervorragend als ideologische Instrumente, weil sich über die Beeinflussung von Emotionen Zukunftseinstellungen und Präferenzen lenken lassen. 
In demokratischen Gesellschaften sollte es eigentlich eine zentrale Aufgabe der Wissenschaftspolitik sein, zwischen der Wissenschaft und der allgemeinen Gesellschaft zu vermitteln. Einerseits sollten gesellschaftliche Erwartungen an die Wissenschaft in entsprechende öffentliche Förderprogramme umgesetzt werden; andererseits sollte auch Breitenaufklärung über wissenschaftliche Potentiale betrieben werden, damit die gesellschaftlichen Erwartungen realistisch sind. Werden diese beiden Kernaufgaben von der Wissenschaftspolitik nicht ausreichend wahrgenommen, wie es heute in vielen Gesellschaften der Fall ist, dann übernehmen andere Gruppen die Vermittlungsrollen. Entsprechend ist heute die Schnittstelle zwischen Wissenschaft und Öffentlichkeit von zahlreichen Gruppen bevölkert, die einerseits Hoffnungen und Ängste in Bezug auf Wissenschaft und Technik gestalten und andererseits ihren spezifischen Klientelinteressen zur Durchsetzung verhelfen. Niemals zuvor ist das so deutlich geworden wie in der Nanotechnologie, denn als programmatische Idee ist Nanotechnologie ein Produkt an der Schnittstelle zwischen Wissenschaft und Öffentlichkeit.

Das liegt historisch insbesondere daran, dass die Nanotechnologie ursprünglich aus dem Bereich der Populärkultur kommt und bis heute auch noch aus dieser Perspektive wahrgenommen wird. Denn bevor Wissenschaftler und Wissenschaftspolitiker versuchten, den Begriff für sich zu vereinnahmen, lebte Nanotechnologie in den beiden miteinander verwobenen Bereichen der amerikanischen Science-Fiction und der futuristischen Technikvisionen sowie deren massenmedialer Verbreitung, was ich im folgenden unter dem Begriff der Populärkultur zusammenfasse. ${ }^{10}$ Die visionäre Technikprophezeiung und das fiktionale Ausmalen technisch veränderter Gesellschaften waren nach dem Zweiten Weltkrieg soweit miteinander verknüpft, dass sie oft von den gleichen Personen betrieben wurden (etwa von Arthur C. Clarke, Robert Heinlein, Isaac Asimov und Ben Nova) und gemeinsame Lobbygruppen zur öffentlichen Förderung der Weltraumbesiedlung gründeten, der Unsterblichkeit mittels Kryonik, der Künstlichen Intelligenz beziehungsweise „Superintelligenz" und so weiter. Das Leitthema der Weltraumbesiedlung, welches zunächst durch die Ängste des Kalten Kriegs und später der Überbevölkerung und Umweltverschmutzung genährt wurde, ließ zahlreiche neue visionäre Techniken ersinnen. Dazu gehörten unter anderem das Recyceln von Nahrung und sonstigen materiellen Dingen auf atomarer Ebene im materiearmen All; das Einfrieren und spätere Auftauen des Raumfahrers auf sehr langen Reisen (Kryonik); der genetische Umbau des Astronauten zur optimalen Anpassung an erdfremde Lebensbedingungen, und die Entwicklung von superintelligenten Computern, um die lange Reise durch den Weltraum oder den Kampf gegen klügere Außerirdische zu steuern. Für all diese Aufgaben wurden seit den 1940er Jahren Technikvisionen zur Gestaltung und Umgestaltung der materiellen Welt auf atomarer Ebene entworfen und zum Teil auch über populäre Filmserien wie Star Trek international popularisiert. 
Mit seinem Buch Engines of Creation (1986) fasste der damalige Weltraumbesiedlungs-Lobbyist Eric Drexler all diese visionären Techniken zur gemeinsamen Vision einer universellen Robotik auf molekularer Ebene zusammen und nannte sie erstmals Nanotechnologie. Drexlers Vision löste umgekehrt eine weitere Welle der Science-Fiction aus sowie die Gründung erlösungsorientierter Technikreligionen, wie die der Transhumanisten, die sich seitdem von der Drexlerschen Nanotechnologie ein breites Heilspektrum erhoffen, indem sie die ehemals für die Weltraumbesiedlung erforderlichen visionären Techniken auf das irdische Leben übertragen, so von körperlicher Selbstperfektionierung bis zur Unsterblichkeit. Sowohl die Science-FictionAutoren mit ihren utopischen und dystopischen Geschichten als auch die Technikvisionäre und technikreligiösen Gruppen, die heute erneut in gemeinsamen Lobbygruppen organisiert sind, haben seitdem vehement versucht, öffentliche Aufmerksamkeit auf sich und die Nanotechnologie zu lenken, indem sie Technikhoffnungen und -ängste der Gesellschaft schürten. Was die Science-Fiction-Autoren nur fiktiv als möglichen gemeinsamen Kraftakt quer zu allen Disziplinen beschreiben und was die Technikvisionäre für die Zukunft als wirklich prophezeien, das fordern die Transhumanisten als Soll ein, indem sie eine disziplinenübergreifende Zusammenarbeit für das Ziel der Menschenverbesserung einklagen, was Wissenschaftspolitiker heute gerne als Konvergenz von Nano-, Bio-, Informations-, und Kognitionstechnologien (NBIC-Konvergenz) bezeichnen (Roco/Bainbridge 2002).

Vermutlich würde man heute kaum noch etwas über Nanotechnologie hören, wenn nicht Anfang 2000 die sogenannte Dotcom-Blase im Investmentbereich geplatzt wäre, so dass Nanotechnologie damals mit ihrer Aura der alles ermöglichenden Wundertechnik als willkommenes next big thing der Investmentbranche beziehungsweise als nächste industrielle Revolution hausieren gehen konnte (Schummer 2004b). Von Investment-Reports über vermeintliche Einführungsbücher in Nanotechnologie bis zu Artikeln in Tageszeitungen und Magazinen, die heute weitgehend ungefilterte Pressemitteilungen sind: Über Nanotechnologie wird in populären Medien hauptsächlich in einem Mix aus visionären und ökonomischen Versprechungen berichtet, wobei wissenschaftlich-technische Gesichtspunkte allenfalls in leicht verdaulichen Häppchen serviert werden. Ob es dabei um Investmentmöglichkeiten in eine Produktionsoder eine Investmentfirma geht oder um eine Wette auf eine Wette auf einen fast beliebigen Mix von Firmen wie in den sogenannten Nano-Indexzertifikaten, es bleibt dabei unerheblich, was Nanotechnologie eigentlich ist, solange man nicht wie Merrill-Lynch wegen Aktienmanipulation verklagt wird, die ihren ehemaligen Nano-Index ohne sachliche Kriterien aufbauten (Reisch 2004). Die Investmentbranche hat damit zusammen mit den weitverbreiten Medien eine wichtige Funktion in der programmatischen Formung von Hoffnungen und Ängsten der Technik eingenommen, in der Nanotechnologie nur ein Mittel und eine willkommene neue Worthülse ist. 
Sowohl die ursprüngliche Science-Fiction als auch die Technikvisionäre kannten dystopische Geschichten zur Nanotechnologie, in der die wundersame Technik entweder von bösen Mächten missbraucht wird oder von alleine außer Kontrolle gerät und sich selbständig macht. Das lieferte die Blaupause eines Großteils dessen, was heute „Nanoethik“ oder „Ethik der Nanotechnologie" genannt wird und in dem sich neben den utopischen und dystopischen Technikvisionären ein breites Spektrum von Geistes- und Sozialwissenschaftlern tummelt, die durch staatliche Fördermittel im Rahmen sogenannter „Begleitforschung“ zur Nanotechnologie angezogen werden und die in den seltensten Fällen Ethiker sind. Diese „Ethik der Nanotechnologie“ trägt nicht unwesentlich dazu bei, dem bunten Strauss von Forschungsbereichen, die heute unter "Nanotechnologie“ rangieren, eine Identität zu verleihen und ihm eine breite Aufmerksamkeit und Wichtigkeit zu sichern, weil Nanotechnologie dadurch so mächtig erscheint, dass sie „ethische Fragen" aufwirft. Durch ihre Fokussierung auf die visionäre Nanotechnologie wird damit jedoch die eigentlich kritische Institution der Ethik zu einem Mittel der Popularisierung breiter Bereiche von Wissenschaft und Technik (Schummer 2008b).

Für die Wissenschaft und die mit ihr verbundene Wissenschaftspolitik ist die visionäre Nanotechnologie der Populärkultur ein nicht unwillkommenes Mittel zur Popularisierung von Wissenschaft gewesen. Zwar sind sich die meisten Wissenschaftler untereinander einig, dass sie weder den Hoffnungen noch den Ängsten der visionären Nanotechnologie gerecht werden können. Doch in ihren öffentlichen Verlautbarungen schmücken prominente Wissenschaftler, Wissenschaftsfunktionäre und Öffentlichkeitsabteilungen von Forschungsinstituten ihre jeweilige Alltagsforschung gerne mit Elementen der Drexler'schen Nanovision (so etwa Smalley 1995, Reed/Tour 2000, Whitesides 2001). So gelingt es, hochspezialisierte Forschungsergebnisse, die nur noch im Kontext spezieller disziplinärer Fragestellungen verständlich sind, als entscheidenden Durchbruch oder gar als Revolution mit gewaltigen gesellschaftlichen Konsequenzen darzustellen.

Der neue Ansatz der Wissenschaftspopularisierung, der sich als Idee hinter der Nanotechnologie verbirgt, konnte nicht nur auf das in der Populärkultur bereits vorgeprägte Verständnis von Nanotechnologie setzen, sondern bedient auch die quasi-philosophische Klaviatur der Ontologie, Erkenntnistheorie, Ästhetik und Ethik. Denn entgegen aller wissenschaftlichen Entwicklungen der vergangenen zwei Jahrhunderte erscheint nun erstens die Welt ontologisch höchst einfach, nämlich aus schlichten Bauklötzen bestehend, die man mit Nanotechnologie beliebig nach Wunsch umordnen und neu zusammensetzen kann. Die im Rahmen von Nanotechnologie neu propagierte Verbildlichung von Wissenschaft mittels mikroskopischer Techniken verhilft zweitens zu der erkenntnistheoretischen Botschaft, dass wissenschaftliche Erkenntnis ein einfacher Abbildungsprozess 
sei, vergleichbar mit dem Fotografieren alltäglicher Gegenstände. Weil die entsprechenden rastersondenmikroskopischen Bilder, die heute zu den Ikonen der Nanotechnologie zählen, nach persönlichem Geschmack mit künstlichen Lichtquellen, Perspektiven, Farben und Glanz versehen werden können, erscheint drittens unter ästhetischen Gesichtspunkten die Natur schön und die Arbeit des Wissenschaftlers vergleichbar mit der eines Künstlers. Und schließlich wird die Nanotechnologie viertens in ein besonderes ethisches Licht gerückt, weil sie aufgrund ihrer vermeintlichen Allmacht Hoffnungen und Ängste der Menschen auslöst.

Die Wissenschaftspolitik hat bezüglich der Nanotechnologie ihre eigentliche Aufgabe der neutralen Vermittlung zwischen Wissenschaft und Gesellschaft nicht nur versäumt, sondern das Thema auch für ihre eigenen Zwecke verwendet. Denn mangels klarer Definitionen konnte Nanotechnologie in ihrer alle Disziplinen vereinnahmenden Weise auch für programmatische Ideen der Wissenschaftspolitik genutzt werden. Dazu gehört vor allem die Idee der Interdisziplinarität und damit die der Auflösung disziplinärer Autonomien, die es den Disziplinen bisher erlaubt haben, ihre eigenen disziplinspezifischen Probleme unter relativer Vernachlässigung allgemeiner gesellschaftlicher Relevanz $\mathrm{zu}$ formulieren. Das Ziel der Interdisziplinarität, wie es über Nanotechnologie wissenschaftspolitisch mehr denn je eingefordert wird, ist somit zugleich der Ruf nach dem Primat der Wissenschaftspolitik in allen strategischen Fragen und Zielsetzungen. Dabei geht es nicht nur um die Zusammenarbeit zwischen den Naturwissenschaften, sondern vor allem auch um ein Zusammenrücken von Natur- und Technikwissenschaften unter gesellschaftlich vorgegebenen Aufgabenstellungen. Insofern sich die Wissenschaftspolitik dabei transhumanistische Ziele der „Menschenverbesserung“ zueigen macht, macht sie sich aus vermeintlichen Allgemeinwohlinteressen auch zum Sprachrohr für Transhumanisten, wie dies in den USA aber auch in Deutschland in ministerialen Verlautbarungen geschieht:

Die Forschungs- und Technologiefelder Nanotechnologie, Biotechnologie, Informationstechnologie und Kognitionswissenschaft werden in Zukunft stärker zusammenwachsen (konvergieren) [...]. Längerfristig könnten mehr und mehr Funktionen des menschlichen Körpers von Artefakten aus dem Bereich der Konvergierenden Technologien übernommen werden und eines Tages in der Lage sein, die sensorischen und mentalen Fähigkeiten des Menschen zu verbessern. (BMBF 2006)

Zusammenfassend lässt sich sagen, dass Nanotechnologie als programmatische Idee nur deswegen so breitenwirksam werden konnte, weil die entsprechenden Ideen ursprünglich aus der Populärkultur stammen und dann insbesondere über die Wissenschaftspolitik auf die Wissenschaft übertragen wurden. Der Ort an dem diese programmatischen Ideen ausgehandelt werden, ist weder die Wissenschaft noch der Hort traditioneller 
Ideologieproduktion, sondern die Schnittstelle zwischen Wissenschaft und Öffentlichkeit, die heute von einer Vielzahl von Gruppen besetzt wird, weil sich darüber gesellschaftliche Hoffnungen und Ängste steuern lassen. Dazu gehören unter anderem Science-Fiction-Autoren, Technikvisionäre, Anhänger von Technikreligionen, Investmentberater, Wissenschafts- und Wirtschaftsmedien, Technikethiker, Wissenschaftsfunktionäre und Wissenschaftspolitiker (Schummer 2004b). Alle diese Gruppen versuchen unter dem Stichwort "Nanotechnologie“ die Rolle von Wissenschaft und Technik insgesamt in der Gesellschaft neu zu positionieren durch die Überwindung einer Reihe von traditionellen Grenzen und Unterscheidungen. Dazu gehören nicht nur die Grenzen zwischen den Disziplinen und zwischen Wissenschaft und Technik, sondern insbesondere auch die zwischen Wissenschaft und anderen gesellschaftlichen Institutionen, zu denen auch Politik, Literatur und Religion gehören sowie zwischen Wissenschaft und ihren Reflexionsinstitutionen, einschließlich Ethik, Ästhetik und Erkenntnistheorie. Da all diese Grenzen im Prozess der Moderne im 19. und 20. Jahrhundert entwickelt wurden, führt Nanotechnologie als programmatische Idee trotz ihrer futuristisch erscheinenden Inhalte gesellschaftlich eher zurück zu vormodernen Strukturen.

\section{Nano als Steigerung der Materialwissenschaften}

In diesem Beitrag habe ich versucht, Nanotechnologie unter drei verschiedenen Gesichtspunkten zu untersuchen, nämlich als Technik oder Forschungsbereich, als soziale Dynamik und als programmatische Idee.

Die Untersuchung des ersten Teils hat gezeigt, dass anders als der Ausdruck suggeriert, Nanotechnologie kaum als eine Technik oder Technologie verstanden werden kann, weil sich dahinter auf wissenschaftlich-technischer Ebene nichts inhaltlich oder strukturell Einheitliches verbirgt, geschweige denn eine neue Technik. Denn weder sind die einzelnen Forschungsbereiche, die zur Nanotechnologie gerechnet werden, neu und inhaltlich miteinander verbunden, noch sind die gängigen Definitionen zureichend, um einen neuen besonderen Bereich herauszuheben. Wer Nanotechnologie dennoch als eine neue Technik bezeichnet, scheint sich eher an ein außerwissenschaftliches Publikum zur richten, das weder wissenschaftlich noch wissenschaftshistorisch genügend gebildet ist, um solche Thesen hinterfragen zu können. Die weitgehende Zurückhaltung von Wissenschaftlern bezüglich der Frage der Neuheit und Einheitlichkeit der Nanotechnologie mag darin begründet sein, dass Wissenschaftler einerseits die Etikettierung ihrer Forschung als eher nebensächlich für die Forschungsergebnisse erachten und andererseits von dem Etikett Nanotechnologie in Form von beträchtlich erhöhten Fördermitteln, Karrierepotentialen und öffentlicher Aufmerksamkeit durchaus profitieren. 
Der Mangel an wissenschaftlich-technischen Kriterien geht Hand in Hand mit der wissenschaftspolitischen Inszenierung von Nanotechnologie seit Januar 2000, die zwar global verläuft, aber weitgehend die Nano-Initiative der USA imitiert einschließlich deren begrifflicher Schwäche, der öffentlichen Rhetorik und des rasanten Wachstums im Budget. Ideengeschichtlich sind dabei Konzepte der amerikanischen Populärkultur der Nachkriegs-ScienceFiction und der Technikvisionäre in wissenschaftspolitische Programme überführt worden, was den Imitationen anderswo oft ungewollt bizarre Kultursprünge abverlangt, so dass man zweifeln könnte, ob die Imitatoren ihr Imitat überhaupt verstanden haben. Denn die Nanoprogramme bedienen in den USA zahlreiche Klientel- und Populärinteressen, die in anderen Ländern nicht immer vorhanden sind - von den großen Bedürfnissen zur Risikokapitalanlage bis zur spezifisch amerikanischen Suche nach Erlösungsheil. Als programmatische Idee wird Nanotechnologie dabei (zumindest im Heimatland) an der Schnittstelle zwischen Wissenschaft und Öffentlichkeit verhandelt und besteht im Versuch, die Position von Wissenschaft und Technik insgesamt in der Gesellschaft neu zu bestimmen. Als soziale Dynamik findet Nanotechnologie aber auch in der Wissenschaft selber statt, indem aus ursprünglichen Lippenbekenntnissen heute ein Prozess der Re-Institutionalisierung der Wissenschaft in allen relevanten Bereichen geworden ist. Dieser Prozess ist sowohl in seinem fast alle Disziplinen und Länder umfassenden Wirkungsbereich als auch in seiner Geschwindigkeit einmalig in der Geschichte der Wissenschaft.

Und doch gibt es Parallelen zu einem früheren Prozess, die keineswegs zufällig sind. Auch die Materialwissenschaften, oder genauer: materials science and engineering (MSE), verdanken sich wesentlich einer Initiative der USWissenschaftspolitik der 1970er Jahre (Bensaude-Vincent 2001, Hentschel 2011). Mit den erklärten Zielen, einerseits die disziplinären Grenzen zwischen Chemie und Physik zu überwinden und andererseits eine stärkere Zusammenarbeit zwischen Natur- und Technikwissenschaften zum Verständnis und der gezielten Herstellung technisch nutzbarer Materialien zu erwirken, war die US-Wissenschaftspolitik erfolgreich bei der Etablierung der neuen Disziplin MSE. Auch dieser Prozess lief weltweit weitgehend durch Imitation der Konzepte und der Etablierung neuer Institute und Departments für dieses Gebiet an Universitäten - zumindest in allen Ländern, die ihre Hochschuletats nicht eingefroren hatten. Und wie Nanotechnologie war auch MSE weder nach allgemeiner Definition noch in ihren speziellen Forschungsprogrammen neu. Vielmehr ging es wissenschaftspolitisch darum, ähnlich wie vermutlich heute bei Nano, mittelfristig neue Forschungsinhalte über den Umweg der sozialen Restrukturierung der Wissenschaft zu beeinflussen. Interessanterweise basiert ein solches wissenschaftspolitisches Programm auf der sozialkonstruktivistischen Idee, dass die wissenschaftlichen Gehalte indirekt über die soziale Struktur der Wissenschaft gesteuert werden könnten. 
Nanotechnologie weist nicht nur inhaltlich einen starken Bezug zu MSE auf, indem die Materialforschung quasi in die Nanotechnologie integriert ist. Sie ist auch in ihren Entstehungsbedingungen sehr ähnlich und erscheint sogar als eine Steigerung der MSE, die an deren wissenschaftspolitischen Erfolg anknüpft. Nicht mehr nur Physik und Chemie, sondern potentiell alle naturund technikwissenschaftlichen Disziplinen sollen in der Nanotechnologie zusammengeführt werden - und wo das nicht ausreicht, müssen Bionano, Nanobio oder gar NBIC greifen (Schummer 2009b). Es kann daher bei Nanotechnologie auch nicht mehr um die Etablierung nur einer neuen Disziplin gehen, sondern um die einer neuen Wissensordnung, die alle disziplinären Wissensgehalte aufeinander bezieht und auf gemeinsame Ziele neu ausrichtet.

Trotz aller Parallelen und Steigerungstendenzen zeigt die Nanotechnologie auch erhebliche Unterschiede zu MSE auf. Denn sie stammt ideologisch aus der Populärkultur, während die Materialforschung im populären Verständnis von Wissenschaft und Technik bisher kaum existierte. Entsprechend gab es bei der Etablierung von MSE praktisch kein visionäres Potential und auch nur eine geringe Popularisierungsbemühung. Die Materialforschung scheint sich eher als innerwissenschaftliches Forschungsfeld etabliert $\mathrm{zu}$ haben, während Nanotechnologie an der Schnittstelle zwischen Wissenschaft und Öffentlichkeit verhandelt wird. Und schließlich ging es bei der Etablierung von MSE lediglich um spezifische Neuausrichtungen und nicht um eine Neudefinition der gesamtgesellschaftlichen Rolle von Wissenschaft und Technik.

Betrachtet man die Nanotechnologie als sozialen Prozess, der sich aus den wissenschaftspolitischen Erfolgen der MSE fortentwickelt hat, dann war ihre Entwicklung in den 1990er Jahren gar nicht so unwahrscheinlich, dass tatsächlich niemand sie hätte voraussehen können. Doch die Unterschiede zu MSE sind so gewaltig, dass bis heute offen ist, wohin die zukünftige Entwicklung geht. Die disziplinären, globalen und sozialen Dimensionen der Nanotechnologie sind zu groß und neuartig, um hier aus historischen Beispielen extrapolieren zu können. Nur soviel ist gewiss: Die Kategorien zur Beschreibung von Wissenschaft und Technik müssen erheblich und unkonventionell erweitert werden, um Prozesse wie Nanotechnologie besser verstehen zu können.

\section{Anmerkungen}

1 Die Tagungsberichte sind online zugänglich und durchsuchbar auf der Website der National Academies Press (http://www.nap.edu, letzter Zugriff: 16.02.2010).

2 Dieser Beitrag fasst einige zentrale Thesen zusammen von Schummer 2009a, insbesondere aus Kapitel 2-4 und 7; für ausführlichere Argumente und Verweise siehe dort. 
3 Schmid u. a. 2003; die eigene Definition revidierend ist Schmid u. a. 2006: 62; siehe dazu auch Schummer 2008a.

4 Siehe etwa den ernüchternden Rückblick auf die Cyclodextrin-Forschung in Szejtli 1996.

5 Für eine entsprechende Graphik und Rhetorik siehe auch The President's Council of Advisors in Science and Technology 2005: 9.

6 Ebd., die Gesamtförderung der Nanotechnologie aus öffentlichen Mitteln wurde für 2001 sogar mit 153,1 Millionen Euro beziffert (S. 18). Vier Jahre später berechnete das BMBF die "Nanotechnologie-Förderung der öffentlichen Hand“ im Jahr 2001 sogar mit 210 Millionen Euro (BMBF 2006).

7 Die einzelnen Länder der EU fördern zwar alle schon länger verschiedene Nanoprojekte, -zentren und -netzwerke, haben sich aber neben dem Rahmenprogramm der Europäischen Kommission eher zögerlich zu eigenen nationalen Initiativen zur Nanotechnologie entschieden, wie Österreich (Austrian NANO Initiative, seit 2004), Niederlande (Nanoned, seit 2005), Frankreich (PNANO, seit 2005) und Deutschland (Nano-Initiative, seit 2006).

8 Die asiatischen Initiativen zur Nanotechnologie sind am besten dokumentiert in dem in Japan herausgegebenen Asia Pacific Nanotech Weekly (http://www.nanoworld.jp/apnw/, letzter Zugriff: 16.02.2010).

9 Die Aufsatzdaten stammen aus dem Science Citation Index Expanded. Dabei wurde die Länderauswahl durch die Affiliation der Autoren beschränkt und die Verwendung von „nano“ für andere gebräuchliche Einheiten (wie etwa Nanosekunde) ausgefiltert. Die Budgetdaten sind den jährlichen Publikationen der NNI entnommen (http://www. nano.gov). Die jährlichen Budgets werden, zumindest als Vorschläge des Präsidenten, bereits Mitte des Vorjahres festgelegt, so dass es für Wissenschaftler eine vorausschauende Orientierung gibt.

10 Zum Folgenden siehe Schummer 2009a, Kap. 5, 6 sowie Schummer 2004b.

\section{Literaturverzeichnis}

Allendorf, Mark, 1998. From Bunsen to VLSI: 150 Years of Growth in Chemical Vapor Deposition Technology. Interface (The Electrochemical Society), 7, Nr. 1, 36-39.

Baird, Davis/Shew, Ashley, 2004. Probing the History of Scanning Tunneling Microscopy. In: Davis Baird, Alfred Nordmann und Joachim Schummer, Hg., Discovering the Nanoscale, Amsterdam: IOS Press, 145-156.

Balzani, Vincenzo/Credi, Alberto/Venturi, Margherita, 2003. Molecular Devices and Machines: A Journey into the Nanoworld, Weinheim: Wiley-VCH.

Barkan, Paul, Hg., 1998. Chemical Research - 2000 and Beyond: Challenges and Visions, New York, Oxford: Oxford University Press.

Bensaude-Vincent, Bernadette, 1998. Eloge du mixte, Paris: Hachette.

Bensaude-Vincent, Bernadette, 2001. The Construction of a Discipline: Materials Science in the United States. Historical Studies in the Physical and Biological Sciences, 31, 223-248.

Bundesministerium für Bildung und Forschung, 2002a. Faktenbericht Forschung 2002, BMBF, Bonn [URL: http://www.bmbf.de/pub/faktenbericht_forschung_2002.pdf (16.02.2010)].

Bundesministerium für Bildung und Forschung, 2002b. Nanotechnologie in Deutschland: Standortbestimmung, BMBF, Bonn [URL: http://www.bmbf.de/pub/nanotechnologie_in_ deutschland-standortbestimmung.pdf (16.02.2010)].

Bundesministerium für Bildung und Forschung, 2006. Nano-Initiative - Aktionsplan 2010, BMBF, Bonn [URL: http://www.bmbf.de/pub/nano_initiative_aktionsplan_2010.pdf (16.02.2010)].

Chemical Abstract Service (CAS), 2008. Statistical Summary, 1907-2007, Columbus OH [URL: http://www.cas.org (16.02.2010)].

Ede, Andrew, 2007. The Rise and Decline of Colloid Science in North America, 1900-1935, Ashgate: Aldershot.

ETC Group, 2003. The Big Down: Atomtech - Technologies Converging at the Nano-scale, ETC., Ottawa [URL: http://www.etcgroup.org (16.02.2010)]. 
Fogelberg, Hans/Glimell, Hans, 2003. Bringing Visibility to the Invisible: Towards A Social Understanding of Nanotechnology, Göteborg University, Göteborg.

Fujita, Yasumoto, 2005. Heterogeneous Scientists Meet in the National Lab: The Atom Technology Project in 1990 s Japan. Unpublished lecture at the conference Nano Before There Was Nano: Historical Perspectives on the Constituent Communities of Nanotechnology, Philadelphia, 18-19. März 2005.

Furukawa, Yasu, 1998. Inventing Polymer Science, Philadelphia: Chemical Heritage Foundation.

Hentschel, Klaus, 2011. Von der Werkstoffforschung zur Materials Science, NTM. Zeitschrift für Geschichte der Wissenschaften, Technik und Medizin, 19, 5-40.

Kawamoto, Hirohisa, 2002. The History of Liquid-crystal Displays. Proceedings of the IEEE, 90, Nr. 4, 460-500.

Kelker, Hans, 1973. History of Liquid Crystals. Molecular Crystals and Liquid Crystals, 21, 1-48.

Lehn, Jean-Marie, 1992. Supramolecular Chemistry - Scope and Perspectives: Molecules Supramolecules - Molecular Devices (Nobel Lecture 1987). In: Tore Frängsmyr und Bo G. Malmström, Hg., Nobel Lectures, Chemistry 1981-1990, Singapore: World Scientific, 444-491.

Mody, Cyrus C. M., 2004. How Probe Microscopists Became Nanotechnologists. In: Davis Baird, Alfred Nordmann und Joachim Schummer, Hg., Discovering the Nanoscale, Amsterdam: IOS Press, 119-133.

Monthioux, Marc/Kuznetsov, Vladimir L., 2006. Who Should Be Given the Credit for the Discovery of Carbon Nanotubes?. Carbon, 44, 1621-1623.

National Science and Technology Council, 1999. Nanostructure Science and Technology: A Worldwide Study, Baltimore: International Technology Research Institute/WTEC [URL: http://www.wtec.org/loyola/nano/ (16.02.2010)].

National Science and Technology Council, Subcommittee on Nanoscale Science, Engineering and Technology, 2000. Nanotechnology Definition. Washington DC, Februar 2000 [URL: http://www.nsf.gov/crssprgm/nano/reports/omb_nifty50.jsp (16.02.2010)].

Nordmann, Alfred, 2006. Unsichtbare Ursprünge - Herbert Gleiter und der Beitrag der Materialwissenschaft. In: Alfred Nordmann, Joachim Schummer und Astrid Schwarz, Hg., Nanotechnologien im Kontext - Philosophische, ethische und gesellschaftliche Perspektiven, Berlin: Akademische Verlagsgesellschaft, 81-96.

Peyerimhoff, Sigrid D., 2002. The Development of Computational Chemistry in Germany. In: K. B. Lipkowitz und D. B. Boyd, Hg., Reviews in Computational Chemistry, Bd. 18, Weinheim: Wiley-VCH, 257-291.

President's Council of Advisors in Science and Technology, 2005. The National Nanotechnology Initiative at Five Years, Washington DC: US Executive Office of the President.

Putin, Vladimir, 2007. Annual Address to the Federal Assembly. 26. April 2007 [URL: http://www.kremlin.ru/eng/speeches/2007/04/26/1209_type70029type82912_125670.shtml (16.02.2010)].

Reed, Mark A./Tour, James M., 2000. Computing With Molecules. Scientific American, 20. Juni [URL: scientificamerican.com].

Reisch, Marc, 2004. A Rose By Any Other Name?. Chemical \& Engineering News, 82, Nr. 16, 8. Roberts, Gareth, Hg., 1990. Langmuir-Blodgett Films, New York: Plenum Press.

Roco, Mihail C./Bainbridge, William S., Hg., 2002. Converging Technologies for Improving Human Performance: Nanotechnology, Biotechnology, Information Technology and the Cognitive Science, Arlington, VA: National Science Foundation.

Schmid, Günter u. a., 2003. Small Dimensions and Material Properties: A Definition of Nanotechnology, Bad Neuenahr: Europäische Akademie [URL: http://www.ea-aw. de/fileadmin/downloads/Graue_Reihe/GR_35_Nanotechnology_112003.pdf (16.02.2010)].

Schmid, Günter u. a., 2006. Nanotechnology: Assessment and Perspectives, Berlin/Heidelberg: Springer. Schummer, Joachim, 2004a. Multidisciplinarity, Interdisciplinarity, and Patterns of Research Collaboration in Nanoscience and Nanotechnology. Scientometrics, 59, 425-465.

Schummer, Joachim, 2004b. Societal and Ethical Implications of Nanotechnology: Meanings, Interest Groups, and Social Dynamics. Techne - Research in Philosophy and Technology, 8, Nr. 2, 56-87 (wiederabgedruckt in Joachim Schummer und Davis Baird, Hg. 2006, Nanotechnology Challenges: Implications for Philosophy, Ethics and Society, Singapore: World Scientific, 413-449).

Schummer, Joachim, 2007. The Global Institutionalization of Nanotechnology Research: A Bibliometric Approach to the Assessment of Science Policy. Scientometrics, 70, 669-692. 
Schummer, Joachim, 2008a. Rezension von G. Schmid u. a.: Nanotechnology: Assessment and Perspectives. NanoEthics, 2, 209-212.

Schummer, Joachim, 2008b. The Popularization of Emerging Technologies through Ethics: From Nanotechnology to Synthetic Biology. Spontaneous Generations: A Journal for the History and Philosophy of Science, 2, Nr. 1, 56-62 [URL: http://jps.library.utoronto.ca/ index.php/SpontaneousGenerations/article/view/3529 (16.02.2010)].

Schummer, Joachim, 2009a. Nanotechnologie: Spiele mit Grenzen, Frankfurt a. M.: Suhrkamp [edition unseld].

Schummer, Joachim, 2009b. From Nano-Convergence to NBIC-Convergence: ,The best way to predict the future is to create it'. In: Mario Kaiser, Monika Kurath, Sabine Maasen und Christoph Rehmann-Sutter, Hg.: Governing Future Technologies: Nanotechnology and the Rise of an Assessment Regime, Dordrecht: Springer, 57-71.

Sherman, John D., 1999. Synthetic Zeolites and other Microporous Oxide Molecular Sieves. Proceedings of the National Academy of Science (PNAS), 96, Nr. 7, 3471-3478.

Simões, Ana/Gavroglu, Kostas, 2001. Issues in the History of Theoretical and Quantum Chemistry, 1927-1960. In: Carsten Reinhardt, Hg., Chemical Sciences in the 20th Century, Weinheim: Wiley-VCH, 51-74.

Smalley, Richard E., 1995. Nanotechnology and the Next 50 Years. Vortrag an der University of Dallas-Board of Councilors, 7. Dezember 1995 [URL: http://smalley.rice.edu/smalley. cfm?doc_id=5336 (16.02.2010)].

Szejtli, József, 1996. Historical Background. In: Jerry L. Atwood u. a., Hg., Comprehensive Supramolecular Chemistry, Bd. 3 (Cyclodextrins), Oxford: Pergamon, 1-3.

Whitesides, George M., 2001. The Once and Future Nanomachine: Biology outmatches futurists' most elaborate fantasies for molecular robots. Scientific American, 16. September (scientificamerican.com).

Woyke, Andreas, 2008. Überlegungen zur Verortung der Nanotechnologie in einem wissenschafts- und technikgeschichtlichen Kontinuum. Berichte zur Wissenschaftsgeschichte, 31, 58-67.

\section{Joachim Schummer}

Richardstr. 100

12043 Berlin

Deutschland

E-Mail: js@hyle.org 